Bull. Austral. Math. Soc.

20D10, 20N05

Vol. 49 (1994) [121-128]

\title{
ON CONNECTED TRANSVERSALS TO ABELIAN SUBGROUPS
}

\section{MarkKo Niemenmaa and Tomas KepKa}

\begin{abstract}
In this paper we investigate the situation where a group $G$ has an abelian subgroup $H$ with connected transversals. We show that if $H$ is finite then $G$ is solvable. We also investigate some special cases where the structure of $H$ is very close to the structure of a cyclic group. Finally we apply our results to loop theory and we show that if the inner mapping group of a finite loop $Q$ is abelian then $Q$ is centrally nilpotent.
\end{abstract}

\section{INTRODUCTION}

If $G$ is a group, $H \leqslant G$ and $A$ and $B$ are two left transversals to $H$ in $G$, then we say that $A$ and $B$ are $H$-connected if $a^{-1} b^{-1} a b \in H$ for every $a \in A$ and $b \in B$. This concept was introduced by the authors in [10] where it was used to characterise multiplication groups of loops. Naturally, connected transversals are interesting in group theory in their own right and the authors continued their investigations in [11] where they managed to prove the following two results: (1) If $G$ is a finite group which has an abelian subgroup $B$ such that there exist $H$-connected transversals $A$ and $B$, then $G$ is solvable. (2) If, in addition, $G=\langle A, B\rangle, B$ is of prime power order and the core of $H$ in $G$ is trivial, then $Z(G) \neq 1$. In the present paper (see Theorem 4.1) we are able to prove (1) also in the case that $G$ is infinite and $B$ is finite (the argument of [11] based on Sylow theorems has to be replaced by other arguments and the use of Zorn's lemma) and we prove (2) without the assumption that $H$ is of prime power order (see Proposition 6.3 and the remark after its proof). We also consider two special cases where $H \cong C_{p}{ }^{(2)}$ and $H \cong C_{p} \times C_{q}{ }^{(2)}$ (here $p$ and $q$ are two different prime numbers). Finally, we prove several consequences of the above results in loop theory. Perhaps the most interesting is the following result: If the inner mapping group of a finite loop $Q$ is abelian, then $Q$ is centrally nilpotent.

\section{Preliminaries}

Connected transversals are defined as in the first section. The core of $H$ in $G$ is the largest normal subgroup of $G$ contained in $H$ and we denote it by $L_{G}(H)$. If $p$ is a prime number then we write $C_{p}$ for the cyclic group of order $p$ and $C_{p}{ }^{(2)}=C_{p} \times C_{p}$. In our proofs we need the following results.

Received 22nd February, 1993

Copyright Clearance Centre, Inc. Serial-fee code: 0004-9729/94 \$A2.00+0.00. 
LEMMA 2.1. If $A$ and $B$ are $H$-connected transversals in $G$ and $L_{G}(H)=1$, then $1 \in A \cap B$ and $N_{G}(H)=B \times Z(G)$.

LEMMA 2.2. Let $H \leqslant G, A$ and $B$ be $B$-connected transversals in $G, C \subseteq$ $A \cup B$ and $K=\langle H, C\rangle$. Then $C \subseteq L_{G}(K)$.

The proofs of results can be found in [10, p.113-114].

LEMMA 2.3. If $H \leqslant G, A$ and $B$ are $H$-connected transversals and $L_{G}(H)=$ 1 , then $Z(G) \subseteq A \cap B$. If $N$ is a normal subgroup of $G$ and $N \subseteq A \cap B$, then $N \leqslant Z(\langle A, B\rangle)$.

THEOREM 2.4. Let $H$ be a cyclic subgroup of a group $G$. Then $G^{\prime} \leqslant H$ if and only if there exists a pair $A, B$ of $H$-connected transversals in $G$ such that $G=\langle A, B\rangle$.

For the proofs, see [12, Lemma 1.4 and Theorem 2.2].

LEMMA 2.5. If $H$ is a cyclic subgroup of a group $G$ and there exist $H$-connected transversals $A$ and $B$ in $G$, then $G^{\prime \prime} \leqslant L_{G}(H)$.

ProOF: This follows directly from [12, Corollary 2.3].

In group theory our notation is standard. In Section 6 we give some applications on loop theory. For the concepts and basic results in loop theory the reader is advised to consult $[1,7,9,10,11]$.

\section{Connected transversals}

In this section we prove several lemmas which will be used later in Section.

LEMma 3.1. Let $K$ be a subgroup of $G$ and let $A$ and $B$ be subsets of $G$ such that $1 \in A \cap B, A B \subseteq B K, A^{-1} B \subseteq B K, B A \subseteq A K$ and $B^{-1} A \subseteq A K$. Then $\langle A, B\rangle \subseteq A K=B K$.

Proof: Now $A \subseteq B K$ and thus $A K \subseteq B K^{2}=B K$. Likewise, $B K \subseteq A K^{2}=$ $A K$, hence $A K=B K=E$. Now denote $F=A \cup B \cup A^{-1} \cup B^{-1}$. Clearly, $A^{-1} \subseteq E$ and $B^{-1} \subseteq E$. Now $A A \subseteq A E=A B K \subseteq B K^{2}=B K=E$ and in a similar way $A^{-1} A \subseteq E$. Thus $F A \subseteq E$ and now $F^{2}=F F \subseteq F E=F A K \subseteq E K=E$. By induction, it is clear that $F^{n} \subseteq E$ and thus $\langle A, B\rangle \subseteq E$.

From now on in this section we assume that $H$ is a subgroup of $G$ and there exist $H$-connected transversals $A$ and $B$ in $G$. We write $E=\langle A, B\rangle$ and if $H \leqslant K$ we denote $C=A \cap K, D=B \cap K, F=\langle C, D\rangle, V=H \cap F$ and $W=H \cap L_{G}(K)$. Naturally, $C$ and $D$ are $B$-connected in $K$ and $V$-connected transversals in $F$. Moreover, $F \leqslant L_{G}(K)$ by Lemma 2.2 and $K=H L_{G}(K)$. Finally, $K / L_{G}(H) \cong H / W$. In the following lemma we prove some technical results. 
LEMMA 3.2. (1) If $V=1$, then $F=C=D \subseteq A \cap B$. (2) If $W=1$, then $F=C=D=L_{G}(K) \leqslant Z(E)$. (3) If $W=1$ and $H<K$, then $Z(E)>1$.

Proof: (1) If $a, b \in C$, then there exist elements $c, d \in C$ and $e \in D$ such that $c^{-1} a b, d^{-1} a^{-1}$ and $e^{-1} a$ are elements of $H$. Clearly, these three elements belong to $V=1$ and thus $a b=c, a^{-1}=d$ and $a=e$. We have shown that $C=D$ is a subgroup, hence $F=C=D \subseteq A \cap B$. (2) Now $W=1$ implies $V=1$ and by (1) $F=C=D$. Clearly, $D=L_{G}(K)$ and $[D, A] \leqslant H \cap D=1$ and $[D, B] \leqslant H \cap D=1$, hence $D \leqslant Z(E)$. (3) This follows directly from (2).

LEMMA 3.3. Let $G=\langle A, B\rangle, L_{G}(H)=1$ and $K=N_{G}(H)$. If $H \cap L_{G}(K)=1$ then $Z(G / Z(G))=1$.

Proof: By Lemma 2.1, $K=N_{G}(B)=H Z(G)$ and since $H \cap L_{G}(K)=1$, it follows that $L_{G}(K)=Z(G)$. By Lemma 3.2, $A \cap K=B \cap K=Z(G)$. Now we write $\bar{G}=G / Z(G)$. Since $L_{\bar{G}}(\ddot{H})$ is trivial, it follows from Lemma 2.3 that $\bar{A} \cap \bar{B}$ contains $Z(G)$. Then denote $E=N_{G}(K)$. Since $\bar{E}=\bar{H} Z(G)$, by Lemma 2.1, we conclude that $\bar{A} \cap \bar{E}=\bar{B} \cap \bar{E}=Z(G)$. But now $\bar{A} \cap \bar{E}=\overline{A \cap E}$, hence $A \cap E=B \cap E$ is a normal subgroup of $G$ and by Lemma 2.3, $A \cap E \leqslant Z(\langle A, B\rangle)=Z(G)$. Thus $Z(G)$ is trivial and the proof is complete.

LEMMA 3.4. Let $H$ be an abelian maximal subgroup of $G$ and assume that $H$ is not normal in $G$ and $1 \in A \cap B$. Then $A Z(G)=B Z(G)$ is a subgroup of $G$ and $G \neq\langle A, B\rangle$.

Proof: It is easy to see that $N_{G}(H)=H$ and $Z(G) \leqslant H$. If $a \in A$, then $b^{-1} a \in H$ for some $b \in B$. Since $a^{-1} b^{-1} a b \in H$ it follows that $b^{-1} a \in H \cap a H b^{-1}=$ $H \cap b H b^{-1}=T$. If $N_{G}(T)=H$, then $b \in H$ and $a=b=1$. If $N_{G}(T)=G$, then $C_{G}(T)=G$, hence $T \leqslant Z(G)$. Thus $a \in B Z(G)$ and we have shown that $A \subseteq B Z(G)$. In a similar way, $B \subseteq A Z(G)$.

If $a \in A$ and $b \in B$, then there exists $c \in B$ such that $c^{-1} a b \in H$. Since $a^{-1} b^{-1} a b \in H$ and $a^{-1} c^{-1} a c \in H$, it follows that $c^{-1} a b a H=c^{-1} a a b H=c^{-1} a c H=$ $a H$. Thus $a^{-1} c^{-1} a b a \in H$, hence $c^{-1} a b \in H \cap a H a^{-1}$. As in the first part of the proof we conclude that $c^{-1} a b \in Z(G)$. This means that $A B \subseteq B Z(G)$ and in the same way $B A \subseteq A Z(G)$.

If again $a \in A$ and $b \in B$, then there exists $c \in B$ such that $c^{-1} a^{-1} b \in H$. Now $c^{-1} a^{-1} b a H=c^{-1} a^{-1} a b H=c^{-1} a c H=a H$, hence $a^{-1} c^{-1} a^{-1} b a \in H$. It follows that $c^{-1} a^{-1} b \in H \cap a H a^{-1}$. As before, $c^{-1} a^{-1} b \in Z(G)$ and thus $A^{-1} B \subseteq B Z(G)$. Of course, we also have $B^{-1} A \subseteq A Z(G)$. By Lemma 3.1, $\langle A, B\rangle \subseteq A Z(G)=B Z(G)$ and now it is easy to see that $A Z(G)$ is a subgroup of $G$. If $G=\langle A, B\rangle$, then $G=A Z(G)$ which means that $H=Z(G)$. Since $H$ is not normal in $G$, we conclude that $\langle A, B\rangle$ is a proper subgroup of $G$. 
LEMMA 3.5. Assume that $G=\langle A, B\rangle, H$ is an abelian subgroup of $G$ and $1 \in A \cap B$. If $R=\bigcap\{K: H<K \leqslant G\}$ and $H<R$, then $H$ is normal in $R$.

Proof: If $H$ is not normal in $R$, then $N_{G}(H)=H$ and $Z(R) \leqslant B$. If $T<H$ and $N_{G}(T)>H$, then $T \leqslant Z(R)$. Now we can proceed as in the proof of Lemma 3.4 and we can show that $G=\langle A, B\rangle=A Z(R)=B Z(R)$. Since $Z(R) \leqslant H$ and $A, B$ are transversals to $H$ in $G$ we conclude that $Z(R)=H$. Thus $H$ is normal in $R$, a contradiction.

\section{MAIN RESULTS}

THEOREM 4.1. Let $H$ be a finite abelian subgroup of a group $G$ such that there exist $H$-connected transversals $A$ and $B$ in $G$. Then $G$ is solvable.

ProOF: We show that there exists a mapping $t: N \rightarrow N$ such that $G^{(t(n))}=1$, where $n=|H|$. From Lemma 2.5, it follows that we can put $t(1)=1$ and $t(2)=t(3)=$ 3. For $n \geqslant 4$ our proof is by induction. We first write $m=\max \{t(k): 1 \leqslant k<n\}$. If $L_{G}(H) \neq 1$, then $H / L_{G}(H)$ and $G / L_{G}(H)$ satisfy the assumptions of our theorem and thus $G^{(m+1)}=1$. Thus we may assume that $L_{G}(H)=1$. This means that $1 \in A \cap B$. Now we divide the proof into three parts.

(1) Assume that $Z(G)=1$ and $H \cap L_{G}(K)>1$ whenever $H<K$. By induction, $G^{(m)} \leqslant L_{G}(K)$. If we write $R=\bigcap\{K: H<K \leqslant G\}$, then $G^{(m)} \leqslant L_{G}(R)$. If $R=H$, then $G^{(m)} \leqslant L_{G}(H)=1$. Thus we assume that $H<R$. Since $Z(G)=1$, we have $N_{G}(H)=H$ by Lemma 2.1. Thus $H$ is not normal in $R$ and $Z(R)<B$. We write $C=A \cap R$ and $D=B \cap R$. Then $C$ and $D$ are $H$-connected transversals in $R$. By Lemma 3.4, $C Z(R)=D Z(R)$ is a subgroup of $R$. It follows that $[C, D] \leqslant$ $C Z(R) \cap H=Z(R)$. Clearly, $Z(R) \leqslant L_{R}(H)$ and if we write $\bar{R}=R / L_{R}(H)$, then $\bar{R}=\overline{C H}$, where $\bar{C}=\bar{D}$ is an abelian subgroup of $\bar{R}$. By the theorem of Ito [6, p.674-675], $\bar{R}^{\prime \prime}=1$, hence $R^{(3)}=1$. Since $G^{(m)} \leqslant R$, we have $G^{(m+3)}=1$.

(2) Now assume that $Z(G)>1$ and $H \cap L_{G}(K)>1$, where $K=N_{G}(H)=$ $H Z(G)$. If we write $\bar{G}=G / L_{G}(K)$, then $\bar{G}^{(m)}=1$, hence $G^{(m)} \leqslant L_{G}(K) \leqslant K$ and $G^{(m+1)}=1$.

(3) Now we write $E=\langle A, B\rangle$ and $\mathcal{A}=\{P \leqslant G: H \leqslant P, P \cap A=P \cap B$ is a subgroup of $\mathcal{Z}(E)\}$. Now $H \in \mathcal{A}, \mathcal{A}$ is ordered by inclusion and clearly we can apply Zorn's lemma. Let $F$ be a maximal element of $\mathcal{A}$. Then $C=F \cap A=F \cap B$ is a subgroup of $Z(E), C$ is naturally an abelian group and $F=C H$, hence $F^{\prime \prime}=1$ by Ito's theorem. If $L_{G}(F) \cap B>1$, then $G^{(m)} \leqslant L_{G}(F) \leqslant F$ and $G^{(m+2)}=1$. Thus we can assume that $L_{G}(F) \cap H=1$. Now we write $\bar{G}=G / L_{G}(F)$ and let $K$ be a subgroup of $G$ such that $F \leqslant K$ and $L_{\bar{G}}(\bar{K}) \cap \bar{H}$ is trivial. Now $\bar{H}=\bar{F} \cong H$ and since $L_{G}(F) \leqslant L_{G}(K)$, it follows that $L_{\bar{G}}(\bar{K})=\overline{L_{G}(K)}$ and $L_{G}(F) \leqslant L_{G}(K) \cap F$. On the 
other hand, from the fact that $L_{\bar{G}}(K) \cap \bar{B}$ is trivial it follows that $L_{G}(F)=L_{G}(K) \cap F$ and thus $L_{G}(K) \cap B=L_{G}(F) \cap B=1$. By Lemma 3.2, $K \cap A=K \cap B=L_{G}(K) \leqslant$ $Z(E)$ and we have shown that $K \in \mathcal{A}$, hence $K=F$. Thus it is clear that $\bar{H} \cap L_{\bar{G}}(\bar{V})$ is not trivial whenever $\bar{H}<\bar{V} \leqslant \bar{G}$. If $Z(G)$ is trivial, then $\bar{G}^{(m+3)}$ is trivial by (1) of this proof, hence $G^{(m+5)}=1$. If $Z(G)$ is not trivial, then $\bar{G}^{(m+1)}$ is trivial by (2) and thus $G^{(m+3)}=1$. From the three parts of our proof it now follows that we can put $t(n)=m+5$. The proof is complete.

We put an end to this section by considering the case where $H$ is elementary abelian of order $\boldsymbol{p}^{2}$.

Lemma 4.2. Let $G=\langle A, B\rangle$ and $H \leqslant G$ such that $H \cong C_{p}^{(2)}$, then $G^{\prime} \leqslant$ $N_{G}(H)$.

Proof: We divide the proof into three parts. (1) If $L_{G}(H)>1$, then $H / L_{G}(H)$ is cyclic and $G^{\prime} \leqslant H$ by Theorem 2.4. Thus we may assume that $L_{G}(H)=1$ (then $1 \in A \cap B)$. (2) Assume now that $Z(G)=1$. If $H<K$, then $H \cap L_{G}(K)>1$ by Lemma 3.2 and $H L_{G}(K) / L_{G}(K)=K / L_{G}(K)$ is cyclic, hence $G^{\prime} \leqslant K$. Thus $G^{\prime} \leqslant R=\bigcap\{K: K>H\}$ and naturally $R$ is normal in $G$. Now $N_{G}(H)=H$ and thus $H<R$ and $H$ is not normal in $R$. This is a contradiction to Lemma 3.5, hence we may assume that $Z(G)>1$. (3) Now consider $T=N_{G}(H)=H Z(G)$. If $H \cap L_{G}(T)=1$, then $L_{G}(T)=Z(G)$ by Lemma 3.2. Thus the core of $T / Z(G)$ in $G / Z(G)$ is trivial and by (2) of this proof $Z(G / Z(G))>1$. On the other hand, this is not possible because of Lemma 3.3. Thus $H \cap L_{G}(T)>1$ and then $H L_{G}(T) / L_{G}(T)$ is cyclic and again by Theorem 2.4, $G^{\prime} \leqslant H L_{G}(T)=T=N_{G}(H)$.

\section{A special case}

In this section we consider the situation where $G$ is a finite group, $H$ is a subgroup of $G$ such that $H \cong C_{p} \times C_{q}{ }^{(2)}$ and there exist $H$-connected transversals $A$ and $B$ in $G$ (here $p$ and $q$ are two different prime numbers).

THEOREM 5.1. If $G$ is a finite group, $G=\langle A, B\rangle$ and $H \cong C_{p} \times C_{q}^{(2)}$, then $L_{G}(H) \neq 1$.

Proof: Assume by induction that $G$ is a counterexample of smallest possible order. Thus $L_{G}(B)=1$. We first show that $Z(G) \neq 1$. If $Z(G)=1$, then $N_{G}(H)=H$ by Lemma 2.1. If $H<K$, then $B \cap L_{G}(K)=R \neq 1$ by Lemma 3.2. If $H / R$ is cyclic, then $G^{\prime} \leqslant H L_{G}(K)=K$ by Theorem 2.4. If $H / R$ is not cyclic, then $H / R \cong C_{q}{ }^{(2)}$ and $G^{\prime} \leqslant N_{G}(K)$ by Lemma 4.2. Thus $G^{\prime} \leqslant \bigcap N_{G}(K)$, where $K$ ranges over all subgroups of $G$ which properly contain $H$. If $T=\bigcap K$, then $G^{\prime} \leqslant N_{G}(T)$. Thus $N_{G}(T)$ is normal in $G$. If $T=H$, then $N_{G}(T)=N_{G}(H)=H$ and $B$ is normal in $G$, a contradiction. Thus $H<T$ and we now have a contradiction to Lemma 3.5. 
Thus we may assume that $Z(G)>1$. Now let $x \in Z(G)$ such that $x \neq 1$ and $|x|=r$, where $r$ is a prime number and consider the group $K=H\langle x\rangle$. If $H \cap L_{G}(K)=1$, then $L_{G}(K) \leqslant Z(G)$ by Lemma 3.2 and, in fact, $L_{G}(K)=(x)$. By induction, the core of $H\langle x\rangle /\langle x\rangle$ in $G /\langle x\rangle$ is nontrivial. Hence we have a normal subgroup $D$ of $G$ such that $\langle x\rangle<D \leqslant H\langle x\rangle$. But now $D \leqslant L_{G}(K)$, a contradiction. Thus $H \cap L_{G}(K)>1$ and the order of $L_{G}(K)$ is one of the following: $p q^{2} r, p q r, q^{2} r, p r$ or $q r$. If $r \neq p$ and $r \neq q$ then we immediately have the characteristic Sylow $p$-subgroup (or the characteristic Sylow $q$-subgroup) in $L_{G}(K)$ and since $L_{G}(K)$ is normal in $G$, we conclude that $L_{G}(H)>1$, which is not possible. Thus $r=p$ or $r=q$. If $r=q$, then the order of $L_{G}(K)$ is one of the following: $p q^{3}, p q^{2}, p q, q^{2}$ or $q^{3}$. In the three first cases we can proceed by using the characteristic Sylow p-subgroup of $L_{G}(K)$. If $\left|L_{G}(K)\right|=q^{2}$ or $=q^{3}$, then $H L_{G}(K) / L_{G}(K)$ is cyclic and by Theorem 2.4, $G^{\prime} \leqslant H L_{G}(K)=K$. Thus $K$ is normal in $G$ and the Sylow p-subgroup of $K$ is normal in $G$, hence $L_{G}(H)>1$, a contradiction. Thus we may finally assume that $r=p$ and $Z(G)$ is a $p$-group. Now the order of $L_{G}(K)$ is one of the following: $p^{2} q^{2}, p^{2} q, p q^{2}, p q$ or $p^{2}$. In the four first cases we have the characteristic Sylow $q$ subgroup in $L_{G}(K)$ and this leads to a contradiction as before. If $\left|L_{G}(K)\right|=p^{2}$, then we write $\bar{G}=G / L_{G}(K)$. Now $\bar{H}=H L_{G}(K) / L_{G}(K)=K / L_{G}(K) \cong C_{q}{ }^{(2)}$. If $L_{\bar{G}}(\bar{H})$ is not trivial, then we can proceed as in the first part of the proof of Lemma 4.2 and we conclude that $G^{\prime} \leqslant H L_{G}(K)=K$. Now $K$ is normal in $G$ and $K$ has the characteristic Sylow $q$-subgroup, hence $L_{G}(H)>1$. Thus we can assume that $L_{\bar{G}}(H)$ is trivial. Then by Lemma $2.1, N_{\bar{G}}(\bar{H})=\bar{H} Z(G)$ and $\bar{H} \cap Z(G)$ is trivial. By Lemma 4.2, $\bar{G}^{\prime} \leqslant N_{\bar{G}}(\bar{H})$, hence $G^{\prime} \leqslant T=N_{G}\left(H L_{G}(K)\right)=N_{G}(K)$ and thus $T$ is normal in $G$. Clearly, $N_{\bar{G}}(H)=\bar{T}$, hence $\bar{H} Z(G)=\bar{T}$. Now we write $N / L_{G}(K)=Z(G)$ and thus $K N=T$. Now by Lemma 2.3, $Z(\bar{G}) \subseteq \bar{A} \cap \bar{B}$ and then $\bar{A} \cap \bar{T}=\bar{B} \cap \bar{T}=Z(G)$. It follows that $(A \cap T) L_{G}(K)=N$. Let $Q$ be the subgroup of order $q^{2}$ in $H$. Now $Q$ is characteristic in $K$, hence $Q$ is normal in $T$. Thus $T=Q \times N$ and $Q \leqslant Z(T)$. On the other hand, $Z(T)=C_{T}(T) \leqslant C_{T}(H) \leqslant N_{T}(H) \leqslant N_{G}(H)=H Z(G)$. Since $T$ is normal in $G$, we know that $Z(T)$ is normal in $G$ and thus $Q \leqslant Z(T) \leqslant L_{G}(H Z(G))$. Since $Z(G)$ is a p-group, it follows that $L_{G}(B Z(G))$ has a characteristic Sylow $q$ subgroup $Q$ and then $Q \leqslant L_{G}(H)$. This is our final contradiction and the proof is complete.

\section{Application to loop theORY}

We say that a groupoid $Q$ is a loop if $Q$ has unique division and a neutral element (thus loops are nonassociative versions of groups). The mappings $L_{a}(x)=a x$ and $R_{a}(x)=x a$ define two permutations on $Q$ for every $a \in Q$ and the permutation group $M(Q)=\left\langle L_{a}, R_{a}: a \in Q\right\rangle$ is called the multiplication group of $Q$. By $I(Q)$ we denote 
the stabilizer of the neutral element and $I(Q)$ is called the inner mapping group of $Q$. It is rather easy to see that the core of $I(Q)$ in $M(Q)$ is trivial and $A=\left\{L_{a}: a \in Q\right\}$ and $B=\left\{R_{a}: a \in Q\right\}$ are $I(Q)$ - connected transversals in $M(Q)$. The concept of the multiplication group of a loop was introduced by Bruck [1] and he used this concept to investigate the structure of loops. Glauberman [3] and [4] studied very thoroughly loops of odd order and Conway [2], Griess [5] and Liebeck [8] have investigated the connection between certain finite simple groups and finite Moufang loops. In [10, Theorem 4.1] Kepka and Niemenmaa gave the following characterisation of multiplication groups of loops.

TheOREM 6.1. A group $G$ is isomorphic to the multiplication group of a loop if and only if there exist a subgroup $H$ satisfying $L_{G}(H)=1$ and $H$-connected transversals $A$ and $B$ satisfying $G=\langle A, B\rangle$.

By combining theorems 5.1 and 6.1 we get the following

Corollary 6.2. If $Q$ is a finite loop then it is not possible that $I(Q) \cong$ $C_{p} \times C_{q}{ }^{(2)}$, where $p$ and $q$ are two different prime numbers.

REMARK. In [10] and [12] it was shown that $I(Q)$ can not be a nontrivial cyclic group. On the other hand, $I(Q) \cong C_{2} \times C_{2}$ is possible as was shown in $[10, \mathrm{p} .120]$.

Our next application deals with the central nilpotency of a finite loop $Q$. For this concept and related results, we advise the reader to consult $[1,9,11]$. We first introduce the following purely group theoretical result.

Proposition 6.3. Let $H$ be an abelian subgroup of a finite group $G$ such that there exist $H$-connected transversals $A$ and $B$ in $G$ and assume that $G=\langle A, B\rangle$. Then $H$ is subnormal in $G$.

Proof: Assume that $G$ is a counterexample of smallest order. If $L_{G}(H)>1$, then $H / L_{G}(H)$ is subnormal in $G / L_{G}(H)$, hence $H$ is subnormal in $G$. Thus we may assume that $L_{G}(H)=1$ (this means that $1 \in A \cap B$.) Then assume that $Z(G)=1$ and let $H<K \leqslant G$. By Lemma 3.2, $H \cap L_{G}(K)>1$. Thus $H L_{G}(K) / L_{G}(K)$ is subnormal in $G / L_{G}(K)$ and since $K=H L_{G}(K)$, we conclude that $K$ is subnormal in $G$. Clearly, $R=\bigcap\{K: H<K \leqslant G\}$ is subnormal in $G$. If $R=H$, then $H$ is subnormal in $G$. If $H<R$, then by Lemma 3.5, $H$ is normal in $R$. Thus we may assume that $Z(G)>1$. Now $H Z(G) / Z(G)$ is subnormal in $G / Z(G)$, hence $H Z(G)$ is subnormal in $G$. But then $H$ is subnormal in $G$ and we are ready.

From the preceding proposition it follows that if $G$ is a finite group, $H<G$ is abelian, $L_{G}(H)=1$ and there exist $H$-connected transversals $A$ and $B$ such that $G=\langle A, B\rangle$ then $Z(G) \neq 1$. This improves the result of Theorem 3.4 in [11] and now proceeding as in Section 4 in [11] we can prove the following interesting result in loop theory. 
Corollary 6.4. Let $Q$ be a finite loop such that the inner mapping group $I(Q)$ is abelian. Then $Q$ is centrally nilpotent.

\section{REFERENCES}

[1] R.H. Bruck, 'Contributions to the theory of loops', Trans. Amer. Math. Soc. 60 (1946), 245-354.

[2] J.H. Conway, 'A simple construction for the Fischer-Griess monster group', Invent. Math. 79 (1985), 513-540.

[3] G. Glauberman, 'On loops of odd order', J. Algebra 1 (1964), 374-396.

[4] G. Glauberman, 'On loops of odd order II', J. Algebra 8 (1968), 393-414.

[5] R.L. Griess, 'A Moufang loop, the exceptional Jordan algebra and a cubic form in 27 variables', J. Algebra 131 (1990), 281-293.

[6] B. Huppert, Endliche Gruppen I (Springer-Verlag, Berlin, Heidelberg, New York, 1967).

[7] T. Kepka and M. Niemenmaa, 'On conjugacy classes in finite loops', Bull. Austral. Math. Soc. 38 (1988), 171-176.

[8] M.W. Liebeck, 'The classification of finite simple Moufang loops', Math. Proc. Camb. Phil. Soc. 102 (1987), 33-47.

[9] M. Niemenmaa, 'Problems in loop theory for group theorists', in Groups St.Andrews 1989 Vol.2, London Math. Soc. Lecture Notes Series 160 (Cambridge University Press, Cambridge, 1991), pp. 396-399.

[10] M. Niemenmaa and T. Kepka, 'On multiplication groups of loops', J. Algebra 135 (1990), 112-122.

[11] M. Niemenmaa and T. Kepka, 'On connected transversals to abelian subgroups in finite groups', Bull. London Math. Soc. 24 (1992), 343-346.

[12] M. Niemenmaa and T. Kepka, 'On loops with cyclic inner mapping groups', Arch. Math. (to appear).

\author{
Department of Mathematics \\ University of Oulu \\ SF-90570 Oulu \\ Finland
}

MFE - UK

Prague

Czechoslovakia 